\title{
Bi-allelic variants in human WDR63 cause male infertility via abnormal inner dynein arms assembly
}

\author{
Shuai Lu ${ }^{1,2,3}$, Yayun Gu ${ }^{2,3}$, Yifei Wu ${ }^{2,3}$, Shenmin Yang ${ }^{4,5}$, Chenmeijie Li, ${ }^{2,3}$, Lanlan Meng ${ }^{6,7}$, Wenwen Yuan ${ }^{2,3}$, Tao Jiang ${ }^{2,3}$,

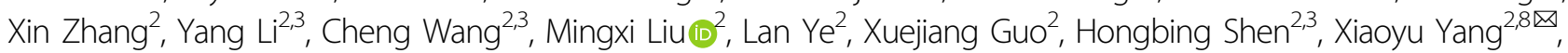 \\ Yueqiu $\operatorname{Tan}^{6,7 凶}$ and Zhibin $\mathrm{Hu}^{1,2 \otimes}$
}

\begin{abstract}
Inner dynein arm (IDA), composed of a series of protein complex, is necessary to cilia and flagella bend formation and beating. Previous studies indicated that defects of IDA protein complex result in multiple morphological abnormalities of the sperm flagellum (MMAF) and male infertility. However, the genetic causes and molecular mechanisms in the IDAs need further exploration. Here we identified two loss-of-function variants of WDR63 in both MMAF and nonobstructive azoospermia (NOA) affected cohorts. WDR63 encodes an IDA-associated protein that is dominantly expressed in testis. We next generated Wdr63-knockout (Wdr63-KO) mice through the CRISPR-Cas9 technology. Remarkably, Wdr63-KO induced decreased sperm number, abnormal flagellar morphology and male infertility. In addition, transmission electron microscopy assay showed severely disorganized " $9+2$ " axoneme and absent inner dynein arms in the spermatozoa from Wdr63-KO male mice. Mechanistically, we found that WDR63 interacted with WDR78 mainly via WD40-repeat domain and is necessary for IDA assembly. Furthermore, WDR63-associated male infertility in human and mice could be overcome by intracytoplasmic sperm injection (ICSI) treatment. In conclusion, the present study demonstrates that bi-allelic variants of WDR63 cause male infertility via abnormal inner dynein arms assembly and flagella formation and can be used as a genetic diagnostic indicator for infertility males.
\end{abstract}

\section{Introduction}

Infertility is a global human health concern and affects approximately $8 \%-12 \%$ of couples around the world ${ }^{1,2}$. Male infertility, which accounts for half of all infertility cases, is generally manifested by reduced sperm count (oligozoospermia and azoospermia), reduced sperm motility (asthenozoospermia), or increased percentage of abnormal sperm morphology (teratozoospermia). Combinations are common, such as "asthenoteratozoospermia" and "oligoasthenoteratozoospermia or OAT syndrome" ${ }^{m, 4}$. The flagellum integrity is essential for normal sperm function

Correspondence: Xiaoyu Yang (yxy1921@163.com) or

Yueqiu Tan (tanyueqiu@csu.edu.cn) or Zhibin Hu (zhibin_hu_njmu@vip.126. com)

${ }^{1}$ Department of Epidemiology, School of Public Health, Southeast University, Nanjing, Jiangsu, China

${ }^{2}$ State Key Laboratory of Reproductive Medicine, Nanjing Medical University, Nanjing, Jiangsu, China

Full list of author information is available at the end of the article and flagellum defects consistently result in male infertility ${ }^{5}$. Multiple morphological abnormalities of the sperm flagellum (MMAF) is one of the most severe asthenoteratozoospermia, which is usually combined with reduced sperm count and is characterized by abnormal flagellar formation, including absent, short, coiled, bent flagellar, and/or irregular caliber, ${ }^{6,7}$.

Genetic factors account for at least 15\% of male infertility, and MMAF is currently one of the most studied male infertility disease with genetic factors ${ }^{8,9}$. With the wide application of high-throughput sequencing technologies, such as whole-exome sequencing (WES), a series of MMAF-associated genes have been identified through genetic studies and animal models. These studies are relevant for MMAF diagnostic value, clinical decision making, and appropriate genetic counseling ${ }^{10-13}$. Owing to high genetic heterogeneity, only approximately $60 \%$ of MMAF-affected cases are caused by previously identified

\section{(c) The Author(s) 2021}

(c) Open Access This article is licensed under a Creative Commons Attribution 4.0 International License, which permits use, sharing, adaptation, distribution and reproduction cc) in any medium or format, as long as you give appropriate credit to the original author(s) and the source, provide a link to the Creative Commons license, and indicate if changes were made. The images or other third party material in this article are included in the article's Creative Commons license, unless indicated otherwise in a credit line to the material. If material is not included in the article's Creative Commons license and your intended use is not permitted by statutory regulation or exceeds the permitted use, you will need to obtain permission directly from the copyright holder. To view a copy of this license, visit http://creativecommons.org/licenses/by/4.0/. 


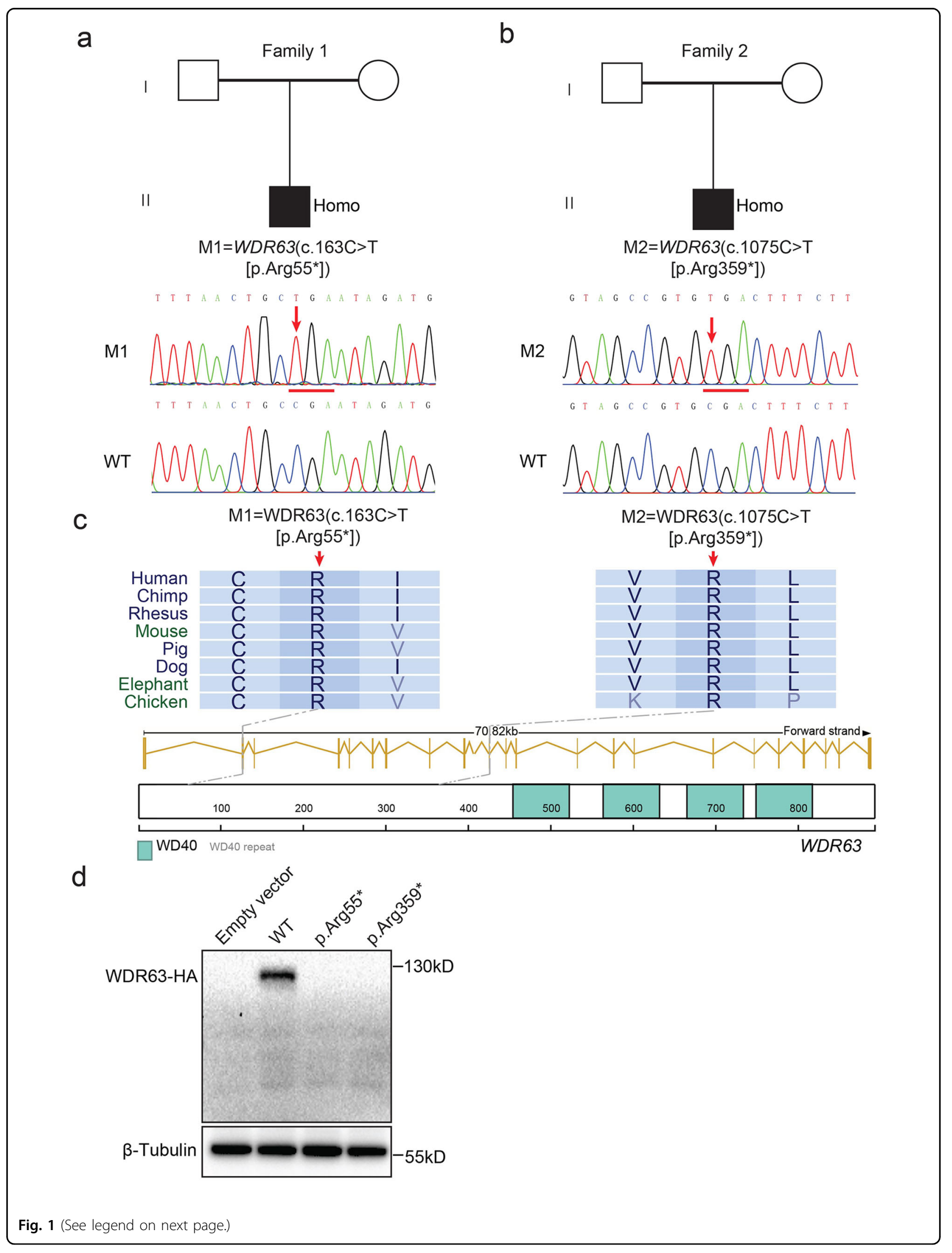


(see figure on previous page)

Fig. 1 Identification of bi-allelic variants of WDR63 in patients with MMAF. a, $\mathbf{b}$ Pedigree of family 1 affected by bi-allelic WDR63 variant that was identified by WES from MMAF-affected men (a). Pedigree of family 2 affected by bi-allelic WDR63 variant that were identified by Sanger sequencing from NOA-affected men (b). Filled black filled squares indicate infertile men in this family. Sanger sequencing results are shown under the pedigrees. The mutated positions are indicated by red arrows. c Schematic representation of the domains of WDR63 and locations of WDR63 variants identified in this study. Sequence alignment shows conservation of the mutated residues across different species according to UCSC genome browser. The green boxes indicate Trp-Asp (WD40) repeat domains as described by the UniProt server. $\mathbf{d}$ WDR63 variants cause the degradation of WDR63 protein. Full-length wild-type and mutant WDR63 cDNA constructs were overexpressed in HEK293T cells followed by immunoblotting analysis. Abbreviations: M1, mutation 1; M2, mutation 2; Homo, homozygous; WD40, Trp-Asp repeat.

Table 1 Bi-allelic WDR63 variants identified in infertility males.

\begin{tabular}{lll}
\hline & M1 & M2 \\
\hline CDNA alteration & C.163C>T & C.1075 C>T \\
Variant allele & Homozygous & Homozygous \\
Protein alteration & p.Arg55* & P.Arg359* \\
Variant type & Nonsense & Nonsense \\
Allele frequency in human population & & $1.3 \times 10^{-04} / 0$ \\
$\quad$ ExAC(all/Asian) & $3.3 \times 10^{-05} / 0.8 \times 10^{-04}$ & $2.9 \times 10^{-04} / 0$ \\
gnomAD (all/Asian) & $1.3 \times 10^{-05} / 0.4 \times 10^{-04}$ & \\
Function prediction & & Damaging \\
SIFT & Damaging & Damaging \\
PolyPhen-2 & Damaging & Damaging \\
Mutation Taster & Damaging & 35 \\
CADD & 37 & \\
\hline
\end{tabular}

NCBI reference sequence number of WDR63 is NP_660155.2.

genetic factors, indicating the necessary to discover new potential MMAF-associated genes.

Motile cilia or flagella are evolutionarily conserved from unicellular protozoa to mammals ${ }^{14}$. Sperm flagella typically comprise a " $9+2$ " axonemal arrangement and a number of multi-protein complexes, including outer dense fiber (ODF), fibrous sheath (FS), nexin-dynein regulatory complex (NDRC), calmodulin-and spoke-associated complex(CSC) and dynein arms $(\mathrm{DAs})^{15}$. Among these protein complexes, the inner dynein arms (IDAs) composed of two heavy chains $1 \alpha \mathrm{HC}$ (DNAH10, in mammals, similarly hereinafter) and $1 \beta$ HC (DNAH2), three intermediate chains, IC140 (WDR63), IC138 (WDR78) and IC97 (LAS1) and several light chains in Chlamydomonas ${ }^{14,16}$. This subcomplex is absolutely required for flagella integrity and assembly, and is responsible for cilia (or flagella) beat frequency and bend formation $^{16}$. For example, functional defective for the WDR78 are completely unable to assemble these structures into the axoneme in vertebrate ${ }^{17}$. Previous studies have shown that variants in IDAs protein complex result in several ciliopathies and male infertility. In particular, bi-allelic in DNAH1 (MIM: 603332), an IDA-associated gene, contributes to MMAF syndrome in human and mice ${ }^{18}$. However, the genetic causes and molecular mechanisms in the other components of IDAs need further exploration.

Here, we identified two bi-allelic variants of WDR63 in both MMAF and NOA-affected cohorts. Furthermore, WDR63 forms a complex with the IDA component
WDR78, and these two proteins co-localized in the sperm flagella. Wdr63-KO impaired the assembly of IDA and caused severely decreased sperm number, sperm motility and abnormal flagellar morphology. Remarkably, intracytoplasmic sperm injection (ICSI) treatment revealed that both Wdr63-KO mice and men harboring WDR63 variants acquired successful clinical pregnancy. Our study suggests that bi-allelic variants of WDR63 can be used as an inherited pathogenic factor and a genetic diagnostic indicator for infertility males.

\section{Results \\ Identification of bi-allelic variants of WDR63 in infertility men}

To identify the potential IDA-associated variants in male infertility, we conducted whole-exome sequencing (WES) analyses in a distinct MMAF cohort depend on variants frequency and functional annotation (minor allele frequency $[\mathrm{MAF}]<0.001$ in the gnomAD database and combined annotation-dependent depletion [CADD] score of $\geq 15$ ) (Supplementary Fig. S1). In the cohort of 243 MMAF-affected Chinese men, we identified a harboring homozygous stop-gain variant (M1: c.163 C > T [p. Arg55*], CADD = 37) in WDR63 (MIM: 617968; NCBI: NM_145172.5) (Fig. 1a and Table 1). Semen parameters of men harboring bi-allelic WDR63 variant was analyzed in the source laboratories according to WHO guidelines $^{19}$. The typical MMAF characteristic was observed in 
the spermatozoa from the men harboring bi-allelic WDR63 variant. Notably, the sperm counts were dramatically lower than the normal reference values (Supplementary Table S1). In addition, we identified an additional bi-allelic variant of WDR63 in 121 non-obstructive azoospermia (NOA) male (M2: c. $1075 \mathrm{C}>\mathrm{T}$ [p.Arg359*], CADD $=35$ ) using Sanger sequencing (Fig. 1b and Table 1 ), indicating that variants of WDR63 might cause the severely decrease in sperm counts.

Human WDR63 contains 23 exons and encodes a predicted 891-amino-acid protein that comprises four TrpAsp (WD40) repeat domains (NCBI: NP_660155.2; UniProt: Q8IWG1). In this study, all of variants in WDR63 are located before the WD40 domain, leading to the loss of WD40 domains in WDR63 protein (Fig. 1c). We overexpressed full-length wild-type and mutant cDNA constructs in HEK293T cells and found the significantly absence of mutant cDNA constructs of WDR63 (Fig. 1d). Based on above results, we speculate that the bi-allelic loss-of-function (LOF) variants of WDR63 is one of the genetic causes of male infertility.

\section{WDR63 is indispensable for male fertility in mice}

To explore the biological function of WDR63 during spermatogenesis, we first characterized tissue-specific distribution of Wdr63 mRNA transcript. Quantitative RT-PCR (q-PCR) analysis revealed that $W d r 63$ transcript was preferentially expressed in testis tissue both in human and mice (Fig. 2a and Supplementary Fig. S2a). We further determined $W d r 63$ transcript levels in mouse testis at different developmental stages. Wdr63 transcript was absent in mouse testis at postnatal day 7 (P7) and P14 (Fig. 2b), when germ cells differentiate to spermatogonia and pachytene spermatocytes, respectively. Further analysis revealed that $W d r 63$ was significantly increased in mouse testis at P21, when round spermatids appear (Fig. $2 b)$.

Considering that mouse WDR63 is highly homologous to human WDR63 protein and primarily expressed in mice testes (Supplementary Fig. S2b), we generated Wdr63-KO mice using CRISPR-Cas9 technology. Sanger sequencing of $W d r 63$ homozygous mutated mice confirmed the presence of a nonsense mutation plus a 2-bp deletion in exon 10, which was predicted to cause premature translational termination (p.Arg362*) (Fig. 2c). Compared with wild-type (WT) male mice, immunoblotting indicated that WDR63 was absent and reduced in homozygous and heterozygous male mice testes, respectively (Fig. 2d). To test male fertility, individual males (wild-type and homozygous) were housed with wild-type females for 2 months. The number of offspring per litter was recorded. Our data revealed that Wdr63-KO male mice were completely infertile, although they were sexually active and produced vaginal plugs in female partners
(Fig. 2e). We also compared testis weights and sizes between WT and Wdr63-KO male mice, but no significant differences were found (Fig. 2f, g). In addition, the same phenotypes were found in $W d r 63$-null male mice with 7-bp frameshift deletion (Supplementary Fig. S3a-j). Consistent with human mutation individuals, these experimental observations indicate that WDR63 is necessary for male fertility in mice.

\section{Wdr63-KO male mice showed severe oligozoospermia combined with MMAF phenotypes}

Having demonstrated the function of WDR63 in male fertility, we next determine the semen characteristics and sperm morphology in Wdr63-KO males. We examined sperm function using the Computer Assisted Sperm Analyzer (CASA) method. Compared with WT male mice, no sperm or very few sperm were detected in the epididymis of homozygotic male mice (Fig. 2h). In addition, the sperm motility and sperm progressive rate was as low as zero in Wdr63-KO male mice (Table 2 and Supplementary Movies S1 and S2). Consistent with the clinical phenotypes of MMAF-affected men with bi-allelic WDR63 variants, the sperm flagella of Wdr63-KO male mice also presented with absent, short, coiled, bent, and/ or irregular shapes (Fig. $3 \mathrm{a}-\mathrm{f}$ and Table 2). Immunofluorescence assays were performed with the cauda epididymis sperm cells from WT and $W d r 63-\mathrm{KO}$ male mice. Immunostaining analysis showed that WDR63 colocalized with Acetyl- $\alpha$-Tubulin (AC-tubulin, a marker of axoneme) along the entire flagella in WT male mice but was almost absent in $W d r 63-\mathrm{KO}$ male mice, indicating that WDR63 is an essential component of axoneme (Fig. $3 g)$.

To investigate the role of WDR63 in different stages of spermatogenesis, we performed Periodic acid-Schiff (PAS) staining on the testes of WT and Wdr63-KO male mice. Gross examination of testes revealed no difference in spermatogonia, spermatocyte and round spermatids between homozygous and wild-type (Fig. 3h). We next counted the number of spermatids and Sertoli cells in stage VII-VIII seminiferous tubules of male mice testis (Supplementary Fig. S4a). Statistical analysis showed no significant difference in Sertoli cells, spermatogonia, spermatocyte and round spermatids between homozygous and wild-type, but the number of elongated spermatids was dramatically decreased in Wdr63-KO mouse (Supplementary Fig. S4b). Besides, the ratios of elongated spermatid and Sertoli cells were decreased in homozygous $(0.21 \pm 0.18)$ compared to wild-type $(3.24 \pm 0.98)$ (Supplementary Fig. S4c). Hematoxylin and eosin (HE) staining of the cauda epididymis from $W d r 63-\mathrm{KO}$ male mice displayed fewer sperm heads than those from WT mice (Fig. 3i).

To further investigate the roles of WDR63 in ultrastructures of spermatozoa in cauda epididymis, we 
a

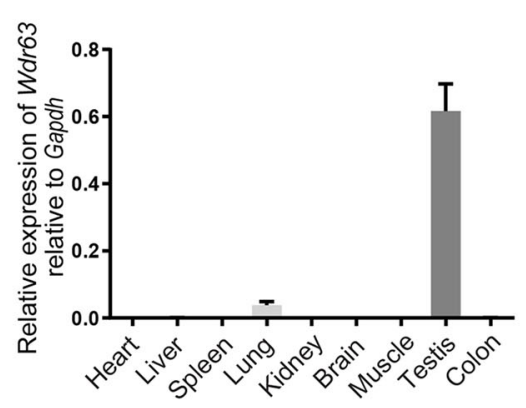

C

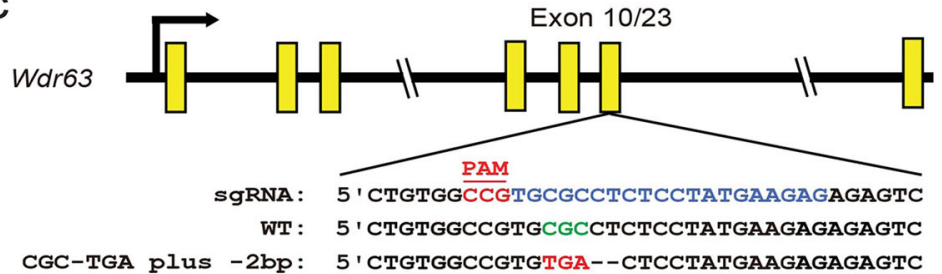

b

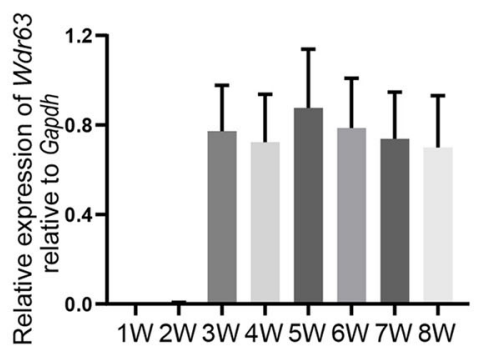

d

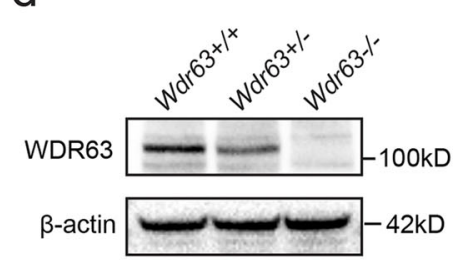

e

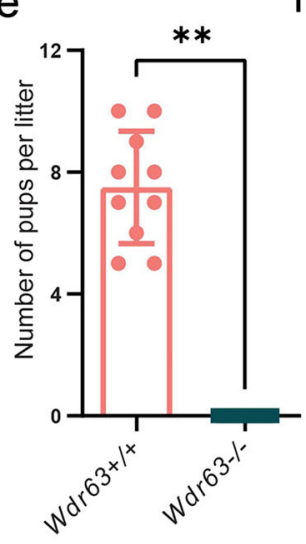

f

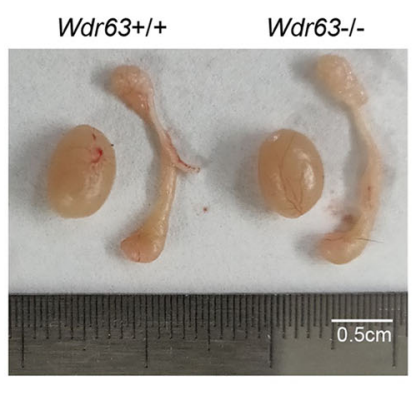

g

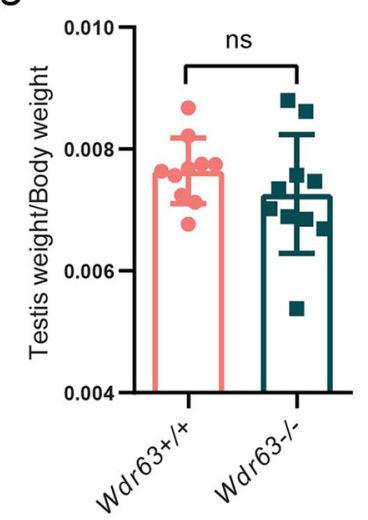

h

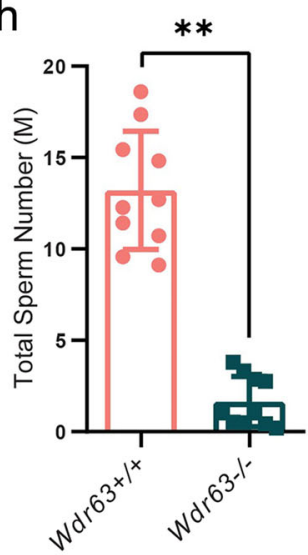

Fig. 2 WDR63 protein is highly expressed in late stages of spermatogenesis and Wdr63-null males are infertile. a Real-time q-PCR for Wdr63 transcripts in various 8-weeks mouse tissues, and Gapdh gene is used as a control. Error bars, SEM $(n=3)$. b mRNA levels of Wdr63 in mouse testis at the indicated time points. Error bars, SEM $(n=3)$. c Schematic diagram of CRISPR/Cas9 strategy for the generation of Wdr63 KO mice. The sgRNA was designed to target exon 10 of the Wdr63, a nonsense mutation (GCG-TGA) plus a 2-bp deletion was obtained. $\mathbf{d}$ Western blot analysis of WDR63 protein in wild-type $\left(\mathrm{Wdr63} 3^{+/+}\right)$, heterozygous $\left(\mathrm{Wdr63} 3^{+/-}\right)$and homozygous $\left(\mathrm{Wdr} 63^{-/-}\right)$mice with a mouse polyclonal antibody raised against WDR63. $\beta$-actin was used as a loading control. e Fertility analysis for adult $W d r 63^{+/+}$and $W d r 63^{-1-}$. f, $\mathbf{g}$ The morphology and testis weight of testes and epididymis from $W d r 63^{+/+}$and $W d r 63^{-/-}$males at 8-week-old. Scale bars, $0.5 \mathrm{~cm}$. $\mathbf{h}$ Total sperm number was dramatically decreased in $W d r 63^{-/-}$ males compared to that of $\mathrm{Wdr}_{\mathrm{C}} 3^{+/+}$males. Abbreviations: $\mathrm{W}$, week; $\mathrm{M}$, million. For $\mathbf{e}$ to $\mathbf{h}, n=10$ and the bars represent means $\pm \mathrm{SEM}$. The statistical analysis was carried out using One-way ANOVA test, ${ }^{* *}$ denotes $P<0.01$; ns, not significant.

conducted Transmission Electron Microscope (TEM) in both WT and Wdr63-KO male mice. Scattered and disorganized axonemal components and cytoplasm residue were observed in the sperm necks and flagella of $W d r 63-$ $\mathrm{KO}$ male mice (Fig. 4a). As show in Fig. 4b, c, crosssections showed severe disorganized " $9+2$ " axoneme combined aberrant inner dynein arm (IDA), outer dynein arm (ODA), outer dense fiber (ODF), fibrous sheath (FS) and nexin-dynein regulatory complex (N-DRC) in the spermatozoa from $W d r 63-\mathrm{KO}$ male mice. These data indicate that WDR63 is required for sperm flagellar formation (Fig. 4d). In summary, we observe WDR63 deficiency caused decreased sperm counts and typical MMAF phenotypes.

\section{WDR63 interacts with WDR78 and their association is necessary for IDA assembly}

WDR63 is the vertebrate orthologue of IC140, a subunit of IDA intermediate chains, is critical for assembly of the IDA complex in Chlamydomonas ${ }^{16}$. Mammalian IDA is a complex structure attached to the peripheral microtubules through docking complexes ${ }^{20}$, and it is essential for bend formation and beating form of cilia and flagella ${ }^{21}$. To characterize the function of WDR63 in vertebrate flagella 
Table 2 Sperm characteristics and flagellar morphology of Wdr63-KO male mice.

\begin{tabular}{lll}
\hline & WT & KO \\
\hline Semen parameter & & $4.83 \pm 3.38^{* *}$ \\
Motility (\%) & $77.79 \pm 8.24$ & $0.71 \pm 0.99^{* *}$ \\
Progressive (\%) & $40.66 \pm 10.30$ & $21.5 \pm 1.49^{*}$ \\
Sperm flagellar morphology ${ }^{a}$ & & $25.84 \pm 5.59^{* *}$ \\
Absent flagella (\%) & $4.05 \pm 1.48$ & $29.12 \pm 5.72^{* *}$ \\
Short flagella (\%) & $0 \pm 0$ & $17.03 \pm 1.86^{* *}$ \\
Coiled flagella (\%) & $0 \pm 0$ & $6.51 \pm 1.02$ \\
Irregular caliber (\%) & $1.53 \pm 0.63$ & $3.15 \pm 1.09$ \\
Bent flagella (\%) & & . \\
\hline
\end{tabular}

${ }^{\mathrm{a}}$ Data represent the means \pm SD of three independent experiments.

${ }^{*} P<0.05,{ }^{* *} P<0.01$.

formation, we first examined whether the protein levels of the other three IDA subunits, DNAH2, DNAH10 and WDR78 were altered in Wdr63-KO testes. Immunoblotting analysis revealed that WDR78 was dramatically decreased, and DNAH2 and DNAH10 were absent in Wdr63-KO. This result indicates that WDR63 is essential for maintaining other components of IDA structure and IDA assembly (Fig. 5a). We next detected the mRNA expression levels of $W d r 63$, Wdr78, Dnah2 and Dnah10 in Wdr63-KO and WT mice. q-PCR analysis revealed that the mRNA expression levels of $W d r 63$ was decreased, but there was no difference in $W d r 78$, Dnah2 and Dnah10 (Supplementary Fig. S5a). To identify protein partners of WDR63, we performed immunoprecipitation with testicular extracts using anti-WDR63 antibodies. Mass spectrometric analysis revealed that WDR78 is the only potential associated protein (Supplementary Data S1). In addition, $W d r 78$ transcript was preferentially expressed in testis, and was significantly increased in mouse testis at P21 (Supplementary Fig. S5b, c). Furthermore, the interaction of WDR63 with WDR78 was verified by coimmunoprecipitation (co-IP) assays (Fig. 5b, c). Immunofluorescence analysis also found that WDR63 co-localization with WDR78, further indicating co-expression between WDR63 and WDR78 (Fig. 5d). Besides, we examined whether WDR63 binds to DNAH2 and DNAH10, which are cooperators of WDR63 in Chlamydomonas ${ }^{16}$. However, immunoblotting followed by coimmunoprecipitation (co-IP) assays demonstrated that WDR63 did not bind to DNAH2 and DNAH10 in mammals (Supplementary Fig. S5d).

To identify the domain required for the interaction of WDR63 with WDR78, we generated HA-tagged full-length WDR63 protein and tagged truncation constructs lacking either the $\mathrm{N}$ terminus (WDR63 $\Delta \mathrm{N}$ ) or the WD40 repeats domain at $\mathrm{C}$ terminus (WDR63 $\Delta \mathrm{C}$ ) (Fig. 5e). co-IP assays revealed that the WDR63-WDR78 interaction was disrupted upon the deletion of the WDR63 C terminus, indicating that the WD40 repeats domain at the $\mathrm{C}$ terminus mediates their interaction (Fig. 5f). These results suggest that WDR63 forms a complex with WDR78 and the assembly of IDA structure requires WDR63 as an essential subunit (Fig. 5g).

WDR63-associated male infertility could be rescued by ICSI

Finally, we aimed to evaluate whether the male infertility induced by WDR63 variants could be overcome by Assisted Reproductive Technology (ART). First, in vitro fertilization (IVF) was utilized to the sperm from WT and Wdr63-KO male mice. The two-cell (2-cell) rates and blastocyst rates were recorded after caudal epididymal sperm fertilized oocytes that were collected from superovulated WT females. However, compared with WT male mice, the rate of 2-cell and blastocyst was zero in $W d r 63$ KO male mice (Fig. 6a). Intracytoplasmic sperm injection (ICSI) has been reported to be efficient for most MMAFassociated patients. To examine whether WDR63associated male infertility could be overcome via ICSI, we conducted experiments using the sperm from WT and Wdr63-KO male mice. Our data show, 2-cell and blastocyst were successfully obtained upon ICSI using the spermatozoa from $W d r 63-\mathrm{KO}$ male mice. Our findings indicated that $W d r 63$-associated $\mathrm{KO}$ male infertility in mice could be overcome by ICSI (Fig. 6b). Consistent with above experimental observations in mice, ICSI using the sperm from Patient-M1 individual was applied and successfully resulted in a live birth. Overall, our data strongly suggest that ICSI could serve as a promising treatment for infertile men harboring bi-allelic WDR63 variants.

\section{Discussion}

In this study, we identified two bi-allelic variants of WDR63 in two male infertility patients. Wdr63-KO male mice showed severely decreased sperm number, sperm 

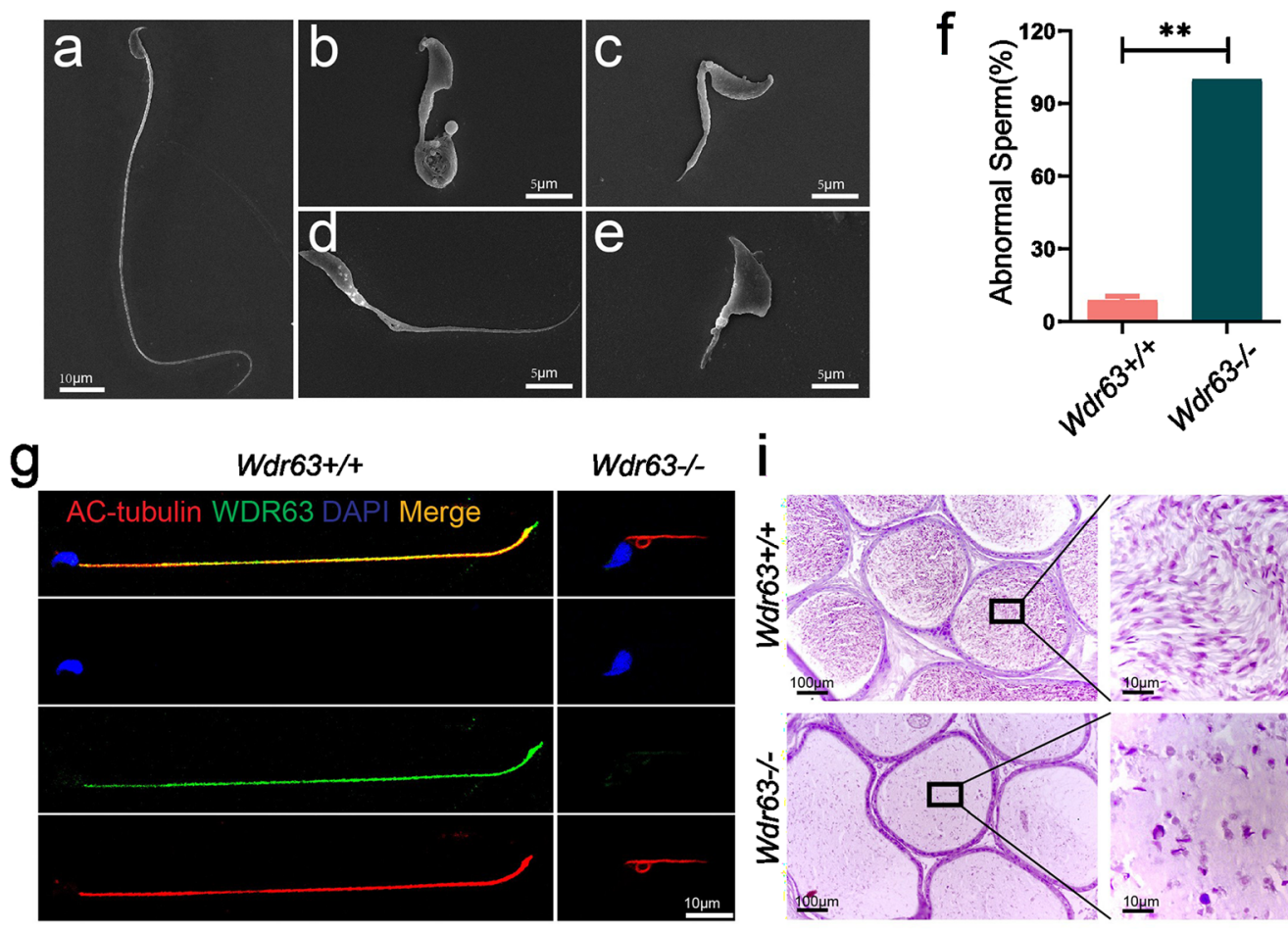

\section{Wdr63-- $\mathbf{i}$}
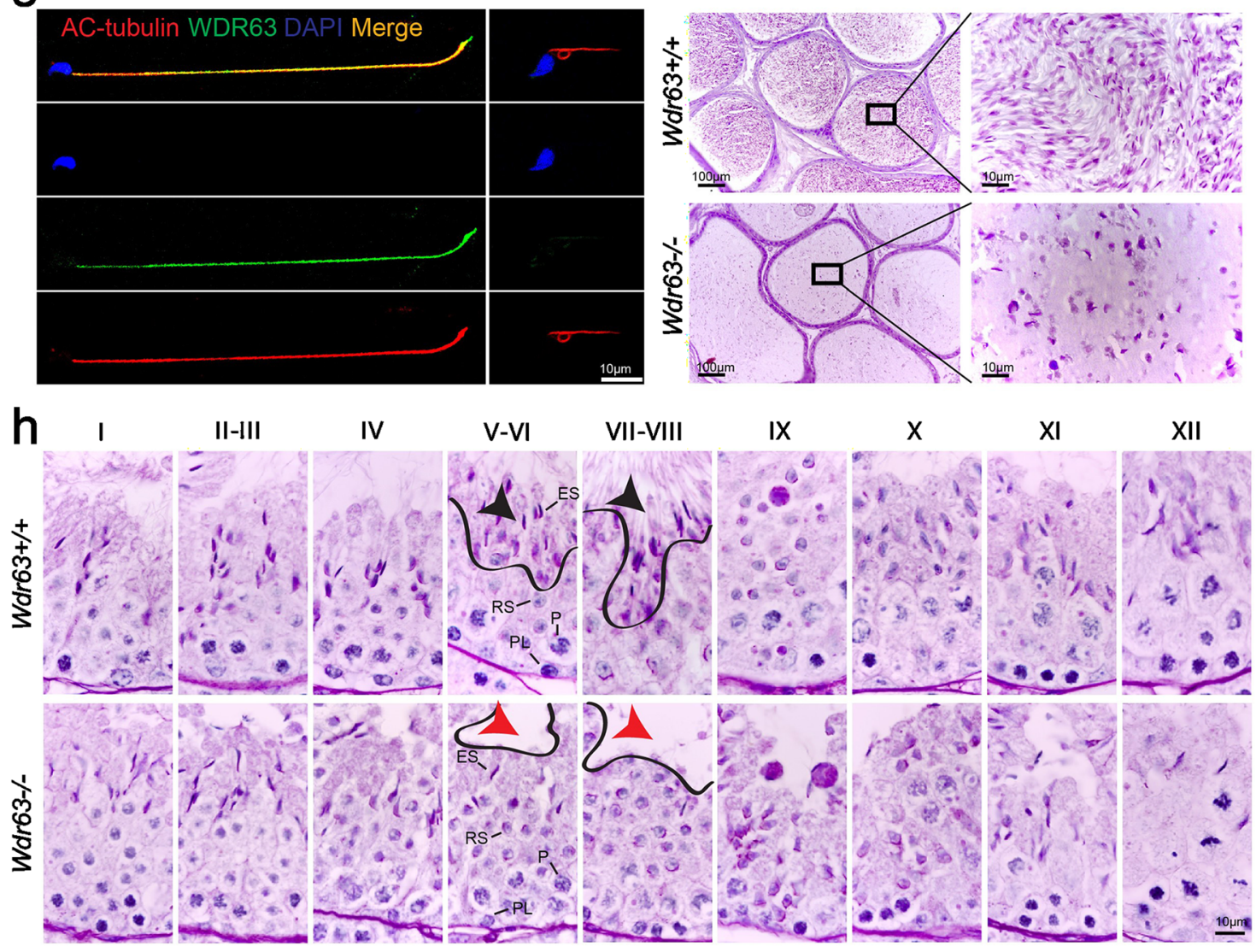

Fig. 3 Wdr63-KO males showed typical MMAF phenotypes. a SEM shows a spermatozoon with normal morphology from the epididymis of Wdr63 $3^{+/+}$males. Scale bars, $10 \mu \mathrm{m}$. b-e SEM shows the spermatozoon of $W d r 63^{-/-}$males presented with MMAF phenotypes, such as short and coiled flagella, flagella of irregular caliber, and other malformations diagnosed as MMAF. Scale bars, $5 \mu \mathrm{m}$. $\mathbf{f}$ Spermatocytograms showing the number of abnormal sperms in WT and homozygous mutant males. $\mathbf{g}$ Immunofluorescent staining of WDR63 (green), AC-Tubulin (red) and DAPI (blue) on the spermatozoa collected from Wdr63 $3^{+/+}$and $W d r 63^{-/}$males. WDR63 localized at entire flagella in WT male mice. Scale bars, $10 \mu \mathrm{m}$. $\mathbf{h}$ Periodic acid-Schiff (PAS) staining of testis sections from adult $\mathrm{Wdr63}^{+/+}$and $W d r 63^{-/-}$mice. Black arrows indicated normal spermatozoon flagella in stage VII-VIII seminiferous tubules of Wdr63 $3^{+/+}$male mice, whereas red arrows indicated the absence of elongated tails in Wdr63 ${ }^{-/-}$male mice. Scale bars, $10 \mu \mathrm{m}$. i HE staining of the cauda epididymis from male mice. Decreased sperm quantity was observed in the epididymis from Wdr63 ${ }^{-/-}$male mice, when compared with that of $W d r 63^{+/+}$male mice. Scale bars, $10 \mu \mathrm{m}$. Abbreviations: PL, preleptotene; P, pachytene; RS, round spermatids; ES, elongated spermatids. For $\mathbf{f}, n=3$ and the bars represent means \pm SEM. The statistical analysis was carried out using one-way ANOVA test, ${ }^{* *}$ denotes $P<0.01$.

motility and abnormal flagellar morphology. In addition, we found that WDR63 binds to WDR78 and is required for IDA assembly and flagella formation.
Notably, the two LOF variants identified in WDR63 were either absent or rare in the human genome datasets archived in the ExAC $\left(\mathrm{MAF}_{\mathrm{M} 1}=3.3 \times 10^{-05}, \mathrm{MAF}_{\mathrm{M} 2}=\right.$ 


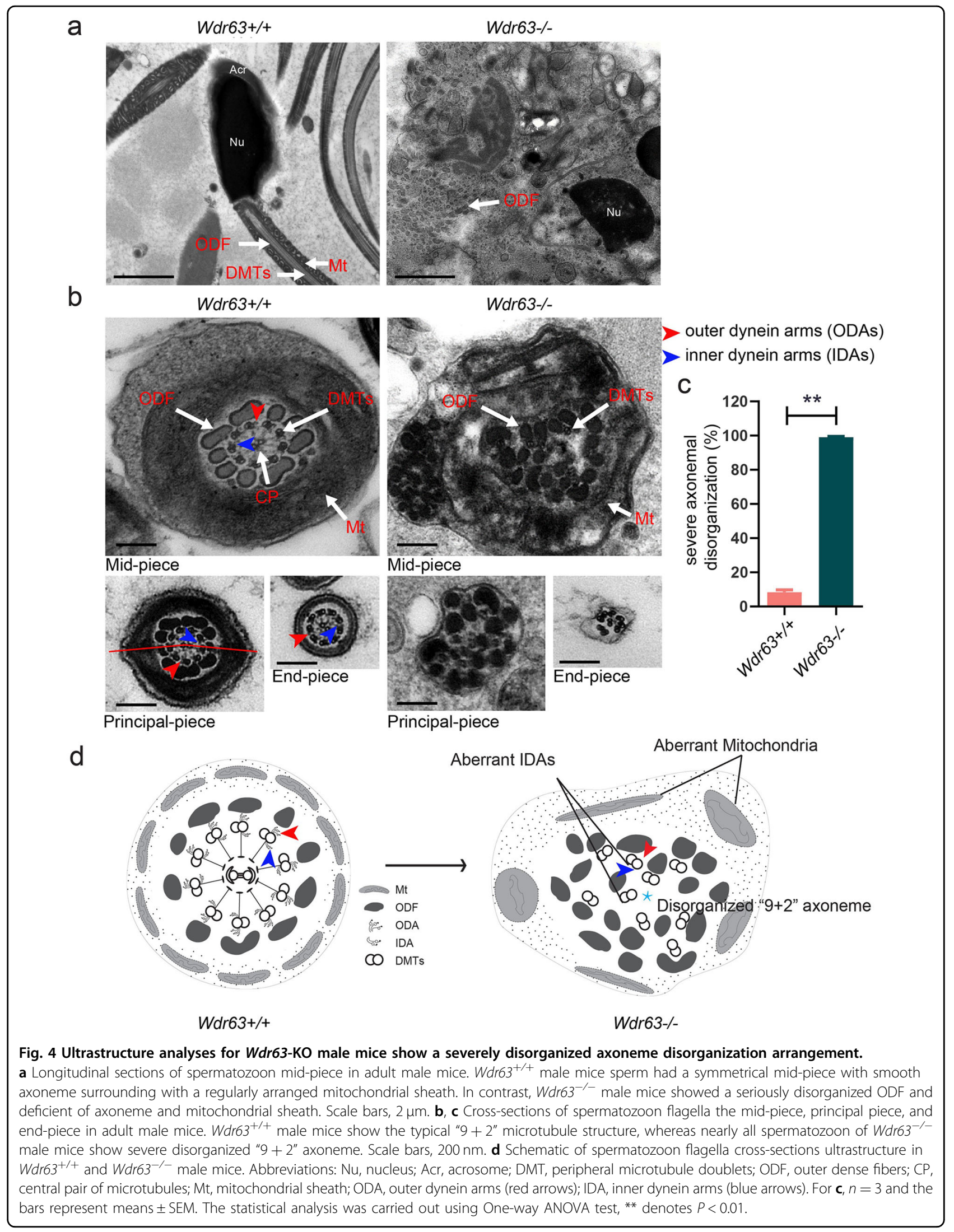




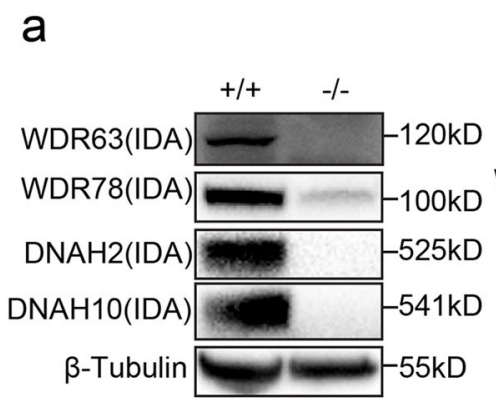

b

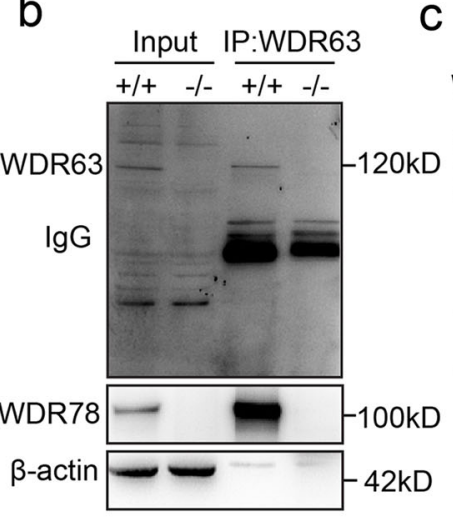

C

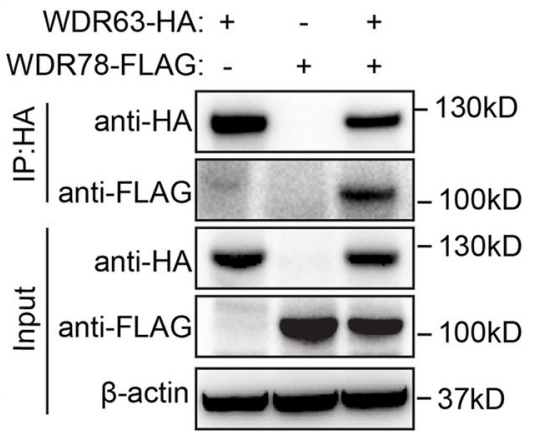

d
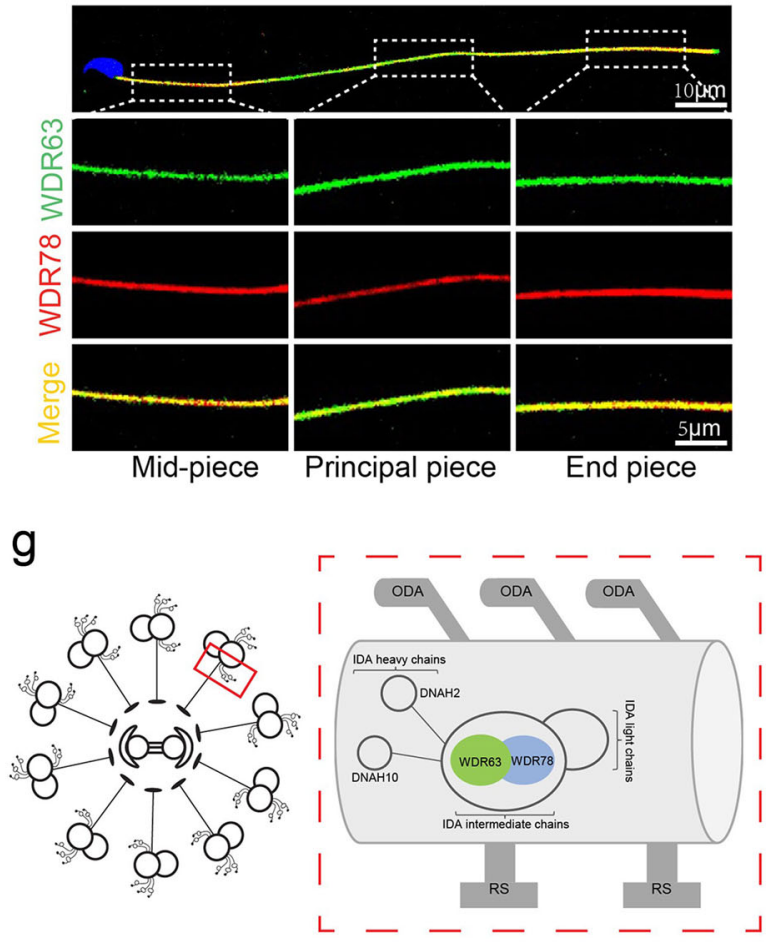

e

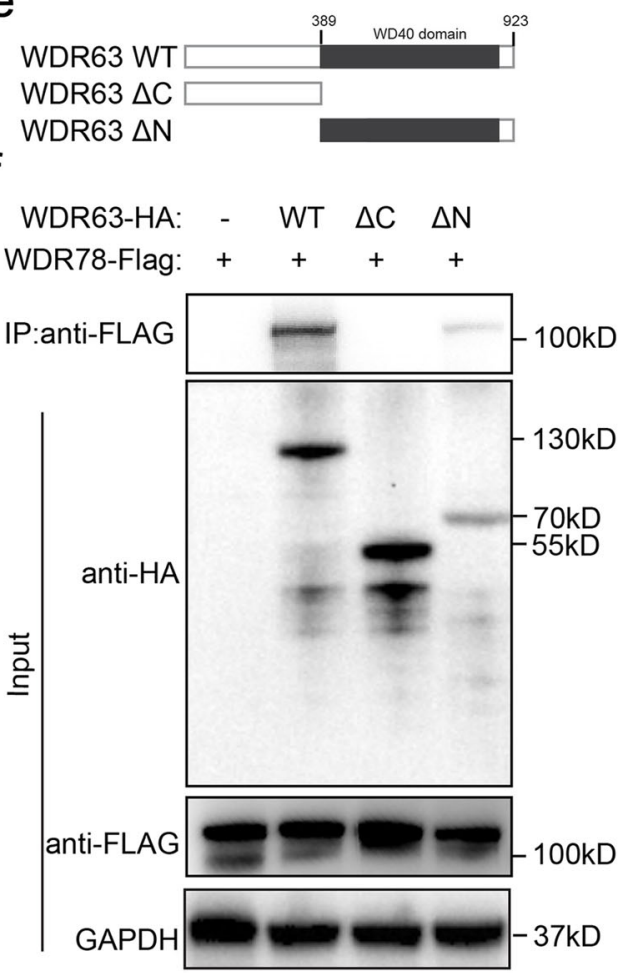

Fig. 5 WDR63 associates with WDR78 and their interaction is necessary for IDA assembly. a Western blot analysis of WDR63, WDR78, DNAH2 and DNAH10 in testes of $\mathrm{Wdr}_{\mathrm{C}} 3^{+/+}$and $W d r 63^{-/-}$male mice. $\mathbf{b}$ Immunoprecipitation with testicular extracts using anti-WDR63 antibodies, Mass spectrometric analysis identified WDR78 is the potential associated proteins. Western blot analysis and confirmed that WDR63 interacts with WDR78. c HA-WDR63 and/or FLAG-WDR78 expression constructs were transfected into HEK293T cells. After $48 \mathrm{~h}$, the cells were collected for

immunoprecipitation analysis with indicated anti-HA and anti-FLAG antibodies. $\mathbf{d}$ Immunofluorescence analysis indicated that WDR63 co-localized with WDR78 in normal spermatozoon. e Schematic diagram of mouse wild type WDR63 (NP_766452.2) and truncated constructs. WD40 repeats domains are marked in a gray box (398-785 aa, UniProt ID: B2RY71). WDR63 $\triangle$ C, deletion of the C terminal domain of WDR63 (398-923 aa); WDR63 $\triangle N$, deletion of the $N$ terminal domain of WDR63 (1-397 aa). f HA-WDR63 WT, HA-WDR63 $\triangle C$ and HA-WDR63 $\triangle N$ were co-transfected with FLAG-WDR78 into HEK293T cells, respectively. After $48 \mathrm{~h}$, the cells were collected for immunoprecipitation analysis with the indicated antibodies. g Schematic of the role of WDR63 interacts with WDR78 in IDAs structure.

$\left.1.3 \times 10^{-04}\right)$ and gnomAD $\left(\mathrm{MAF}_{\mathrm{M} 1}=1.3 \times 10^{-05}\right.$, MAF $_{\mathrm{M} 2}=2.9 \times 10^{-04}$ ) databases without homozygous (Table 1). However, it is unclear if WDR63 variants inherited from M1 and M2 parents or spontaneous because no DNA samples of M1 and M2 consanguineous parents was available. WDR63 encodes a predicted 891- amino-acid protein that comprises four Trp-Asp (WD40) repeat domains (398-785 aa, Uniprot ID: B2RY71). In the present study, both of the two variants in WDR63 are located before the WD40 domain (p.Arg55* and p. Arg359*), leading to the loss of WD40 domains in WDR63 protein. The basic common function of all 


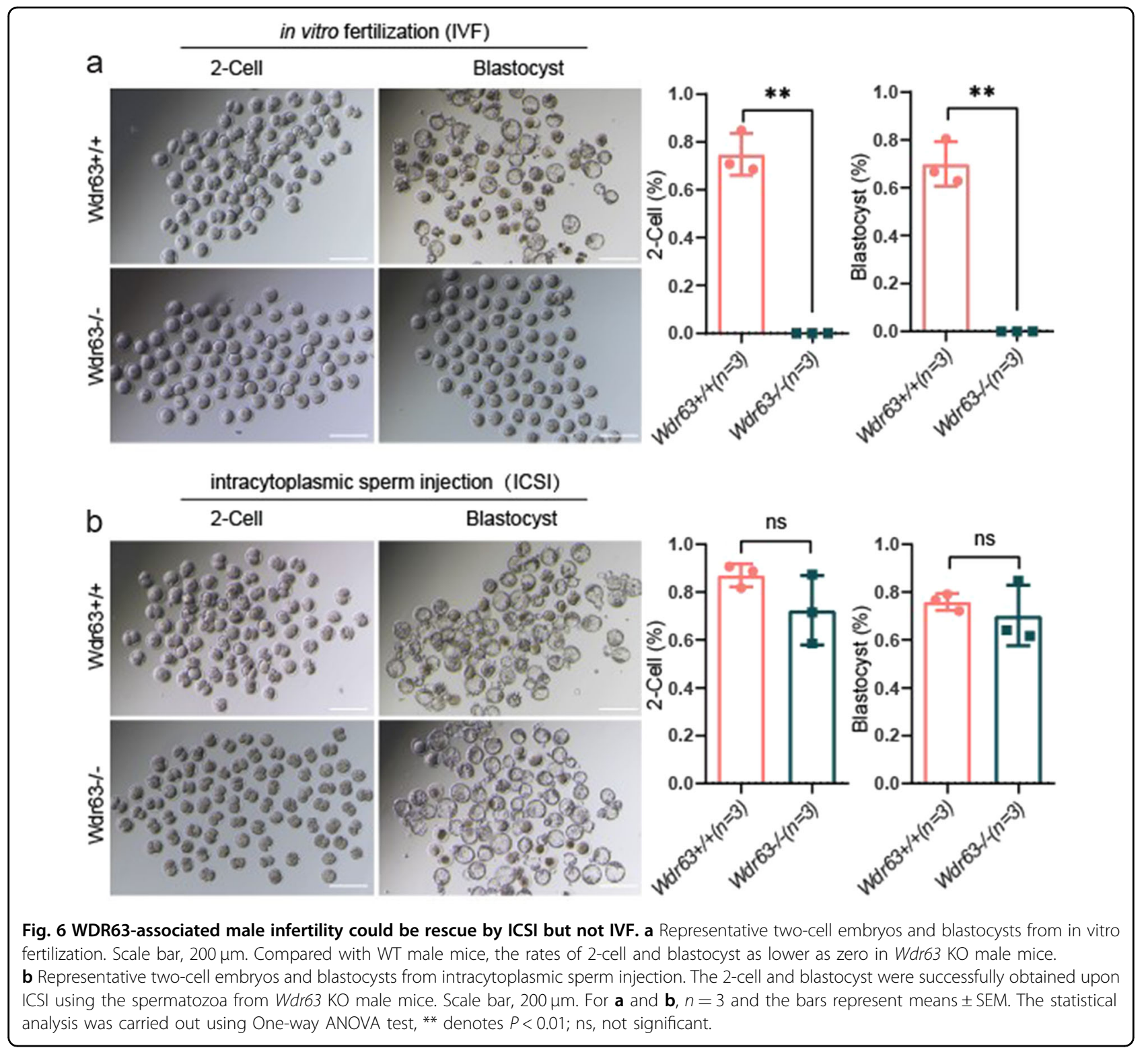

WD40-repeat proteins is to coordinate the assembly of multi-protein complex, where the repeating units act as a rigid scaffolds for protein interactions ${ }^{22}$. The complex structure of sperm flagella contained a series of WD40domain-containing proteins ${ }^{1,23}$. These proteins including CFAP43 (also known as WDR96, MIM: 617558), CFAP44 (also known as WDR52, MIM: 617559) and WDR66 (MIM: 618146) are associated with MMAF and their biallelic variants can reduce sperm motility and cause male infertility $^{24-26}$. In our study, we found that lacking WD40 repeats domain affects the binding of WDR63 and WDR78. These findings indicated that WD40-domaincontaining proteins could play an important role in ciliaand flagella-associated functions. Therefore, we speculated that the lack of the WD40 domain reduced by the
LOF variants in WDR63 may be an important factor in our MMAF patients.

In this study, we demonstrated that $W d r 63-\mathrm{KO}$ male mice is completely sterility. In addition, we noticed that a

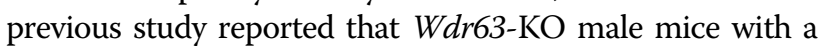
$472 \mathrm{bp}$ deletion spanning exon 2 and exon 3 exhibited normal fertility ${ }^{27}$. Further analysis of $W d r 63$ gene transcripts, we found that $W d r 63$ produces two distinct $W d r 63$ mRNA isoforms differing in their transcriptional initiation, named long isoform (NM_172864.3) and short isoform (XM_017319579.3) (Supplementary Fig. S6a). Our data indicated that both of the transcripts were expressed in wild-type mice testes, suggesting that two transcripts have the potential to be translated into proteins (Supplementary Fig. S6b, c). Thus, we engineered a sgRNA target on exon 
10 of $W d r 63$, which located at common regions both of the transcripts (Supplementary Fig. S6a). Besides, the off-target evaluation of CRISPR-Cas9 suggested that the two genotypes Wdr63-KO mice were successfully on-target (Supplementary Fig. S7). Therefore, we speculate that the phenotype of Wdr63-KO male sterility can only be caused when two transcripts are absent at the same time.

WDR63 and WDR78 are subunits of IDA intermediate chains in mammalian. The IDAs are highly conserved from unicellular protozoa and sperm to the mammalian ciliated epithelia of the respiratory and reproductive tracts $^{21}$. Most studies have shown IDAs are both necessary and sufficient to cilia and flagella bend formation and beating form in Chlamydomonas ${ }^{16}$. Notably, variants or knockdown of IDA complex proteins result in severe asthenoteratozoospermia and/or primary ciliary dyskinesia (PCD) in mammalian. For instance, the first report MMAF-associated gene DNAH1 is component of monomeric IDA heavy chains, and variants in DNAH1 result in damage of IDA without $\mathrm{PCD}^{18}$. Furthermore, variants in IDA-associated proteins DNAH2 (MIM: 603333), DNAH6 (MIM: 603336) can cause severe sperm flagella defects that damage sperm motility ${ }^{28,29}$. In our study, WDR63 is the vertebrate orthologue of IC140, a subunit of IDA intermediate chains in Chlamydomonas, indicating its IDA location and potential function in human and mice sperm flagellar. Previous studies revealed that IC140 is essential for the flagellar assembly of Chlamydomonas, but it is poorly known in mammalian ${ }^{30,31}$. We found that the expression of DNAH10, DNAH2 and WDR78 were significantly decreased in $W d r 63-\mathrm{KO}$ mice, demonstrating the important roles of WDR63 in flagellar assembly in mammalian. Notably, we performed immunoprecipitation with testicular extracts using anti-WDR63 antibodies, mass spectrometric analysis only identified WDR78 is the potential associated proteins. Further co-IP experiments confirmed that WDR78 is associated with WDR63. Immunofluorescence analysis also found the colocalization of WDR63 and WDR78. These experimental observations indicate that WDR63 interacts with WDR78 and is necessary for IDA assembly.

Presently, assisted reproductive technology (ART), such as IVF and ICSI, expanded opportunities for infertile couples $^{32}$. Previous studies have suggested that MMAFaffected men harboring DNAH1, DNAH8 or TTC29 (MIM: 618735) variants could have success clinical outcomes following ICSI ${ }^{18,33,34}$. In contrast, CEP135 (MIM: 611423) and DNAH17-associated MMAF men have a failed pregnancy ${ }^{2,35}$. In this study, although IVF experiments failed to obtain the two-cell embryo and blastocyst from $W d r 63-\mathrm{KO}$ male mice, the rate of two-cell embryo and blastocyst with ICSI experiments were normal in Wdr63-KO male mice compared with WT male mice. This experiment suggesting that male infertility of
Wdr63-KO mice could be overcome by ICSI. Furthermore, MMAF-affected men who harboring WDR63 variants underwent ICSI treatment acquired successful clinical pregnancy and obtained a biological offspring. Therefore, our findings demonstrate that ICSI can be recommended for WDR63-associated MMAF.

In conclusion, we identified that bi-allelic variants of WDR63 cause male infertility via abnormal inner dynein arms assembly and flagella formation. Our finding will be informative for clinical decision making and appropriate genetic counseling of MMAF.

\section{Materials and methods \\ Human samples}

The first cohort of 243 MMAF-affected Chinese men recruited from the Reproductive and Genetic Hospital of CITIC-Xiangya (Changsha, China). Subjects with evident PCD-related symptoms, such as recurrent airway inflammation, bronchiectasis, and otitis media, were excluded. Patients presenting with other causes of infertility, such as reproductive malformation, drug use, and exposure to gonadotoxic factors, were also excluded. The second cohort of 121 NOA-affected Chinese men was enrolled from the First Affiliated Hospital of Nanjing Medical University (Nanjing, China). Those with a history of cryptorchidism, vascular trauma, orchitis, obstruct of the vas deferens, abnormalities in chromosome number or microdeletions of the azoospermia factor region on the $\mathrm{Y}$ chromosome were excluded from the study. This study was approved by the institutional review boards at all the participating institutes. Signed informed consents were obtained from all subjects participating in the study.

\section{Semen analysis and sperm morphological analysis}

Semen analysis was performed in the source laboratories during routine biological examination of the individuals according to the World Health Organization (WHO) guidelines ${ }^{19}$. Semen samples from the men harboring WDR63 variants were collected through masturbation after 2-7 days of sexual abstinence and evaluated after liquefaction for $30 \mathrm{~min}$ at $37^{\circ} \mathrm{C}$. Analyses of semen volume, sperm concentration, and motility were carried out and replicated in the source hospitals during routine examination.

Semen samples from mice were collected from the cauda epididymides obtained through dissection of adult male mice and incubated in human tubal fluid (HTF) medium (Millipore, Cat. \# MR-070-D) supplemented with $10 \% \mathrm{FBS}$ at $37^{\circ} \mathrm{C}$ for $5 \mathrm{~min}$. Sperm count and motility assessment were performed using the Computer Assisted Sperm Analyzer (CASA) system.

\section{Whole-exome sequencing (WES) and Sanger sequencing}

We performed a two-stage sequencing analysis. Genomic DNA was isolated from peripheral-blood samples of the 
subjects using a whole blood DNA purification kit (Qiagen, Hilden, Germany). At the first stage, Whole-exome sequencing (WES) was performed on MMAF-affected subjects as previously described ${ }^{4}$. Briefly, genomic DNA sequencing libraries were prepared with the Nextera Rapid Capture Exome sample preparation kit, targeting $>214,000$ coding exons and following the protocol provided by the manufacturer (Illumina). Nextera Rapid Capture Exome libraries were sequenced on HiSeq2000 with 100-bp pairedend reads. FastQ files were subsequently trimmed and aligned to the human reference genome (hg19, GRCh37) with the Burrows-Wheeler Aligner (BWA, v.0.6.1) ${ }^{36}$. For each sample, the mapping efficiency of generated WES reads was $99 \%$ and the coverage of exonic regions with $\geq 10$ reads was $93 \%$. Single-nucleotide substitutions and small indel variants were called with Genome Analysis Toolkit 1.6-7-g2be5704 (GATK) ${ }^{37}$. Variant sites $>10$ bp away from the nearest exome sequence capture target or with low quality score $(<\mathrm{Q} 20)$ were filtered out. ANNOVAR was performed for functional annotation through a variety of databases, such as ExAC and gnom $\mathrm{AD}^{32}$. After filtering, the retained variants were submitted to PolyPhen-2, SIFT, Mutation Taster and CADD for functional prediction ${ }^{38-40}$. The second stage, candidate pathogenic gene from WES sequencing were validated by Sanger sequencing with another $121 \mathrm{NOA}$-affected subjects. PCR amplification was performed with Dyad Polymerase (Bio-Rad Laboratories). DNA sequencing of PCR products was conducted on an ABI377A DNA sequencer (Applied Biosystems). The primers for PCR are listed in Supplementary Table S2.

\section{Screening process of harboring variants in WDR63 gene}

To identify the potential MMAF-associated variants in male infertility, we first defined testis-high expression genes (THG) according to our previous study ${ }^{41}$. Briefly, a total of 20,719 unique genes with expression abundance data (FPKM) from three datasets (GTEx, Illumina Human Bodymap and NJMU-seq) were included in the analysis after integration. As a result, we identified 1606 THGs characterized with Specificity measure values $>0.8$ $\left(\mathrm{SPM}_{\mathrm{GTEx}}>0.8, \quad \mathrm{SPM}_{\mathrm{HBM}}>0.8, \quad \mathrm{SPM}_{\mathrm{NJMU}}>0.8\right)$ and highly mRNA expression (FPKM $>5)$. Base on above results, we identify 539,641 variants from 243 MMAFaffected Chinese men with whole-exome sequencing (WES) datasets. According to previous pedigree analyses, MMAF has been assumed to follow an autosomal recessive inheritance ${ }^{25,34}$. Therefore, we mainly focused on biallelic rare variants depend on variants frequency and functional annotation, and variants minor allele frequency $(\mathrm{MAF}) \geq 0.001$ in the gnomAD database and combined annotation-dependent depletion (CADD) score of $<15$ were filtered out. Nonsense, frameshift, and essential splice-site variants were preferred. Missense variants predicted to be deleterious simultaneously by the bioinformatics tools of SIFT, PolyPhen-2, and/or Mutation Taster were also included for further evaluation. Finally, 18 candidate genes with 43 bi-allelic loss-offunction variants were retained and validated by Sanger sequencing. Among these genes, CFAP65, CFAP47, FSIP2, CFAP43 and TTC21A have been reported in other study $^{4,11,22,25,42}$. Inner dynein arm (IDA), composed of a series of protein complex, is necessary to cilia and flagella bend formation and beating ${ }^{16}$. Previous studies indicated defects of IDA protein complex result in multiple morphological abnormalities of the sperm flagellum (MMAF) and male infertility ${ }^{29,43,44}$. Thus, we selected the IDAassociated protein, WDR63, from remaining 13 candidate genes for the follow-up study (Supplementary Fig. S1).

\section{Generation of Wdr63 knockout mouse models}

Wdr63 knockout mouse model was created by the animal center of Nanjing Medical University (Nanjing, China). Briefly, single guide RNA (sgRNA: CCGTGCGCCTCTCCTATGAAGAG) was designed to target exon 10 of the $W d r 63$. The oligonucleotide used to generate the sgRNA expression plasmid was annealed and cloned into the BsaI site of pGL3-U6-sgRNA-PGK-puromycin (Addgene 51133). Transcription and microinjection of CRISPR/Cas9 was performed in vitro ${ }^{45}$. Briefly, the Cas9 plasmid (pST1374-Cas9-N-NLS-Flag-linker, Addgene 44758) was linearized with AgeI and transcribed using the T7 Ultra Kit (Invitrogen), followed by purification using RNeasy Mini Kit (Qiagen). pUC57-sgRNA expression vectors were linearized by DraI and transcribed using the MEGAshortscript Kit in vitro (Invitrogen). sgRNAs were purified by MEGAclear Kit (Invitrogen). Mixture of Cas9 mRNA (20 ng/ $\mu \mathrm{l})$ and two sgRNAs $(5 \mathrm{ng} / \mu \mathrm{l}$ each) were injected into the cytoplasm and male pronucleus of the zygote by electroporation. Embryos were implanted into pseudo-pregnant C57BL/6J females according to standard procedures. Founder mice were backcrossed to C57BL/6 J. The off-target evaluation of CRISPR Cas9 suggested that the Wdr63-KO mice were successfully on target (Supplementary Fig. S3). All animal studies were approved by the Institutional Animal Care and Use Committee of Nanjing Medical University, Nanjing, China. The primer used for Wdr63-KO mouse genotype analysis are listed in Supplementary Table S3. Primers used for off-target evaluation are listed in Supplementary Table S4.

\section{WDR63 combinant protein and antibody generation}

The full-length coding sequence of the Wdr63 was subcloned into a pReceiver-B01 (OmicsLinkTM) expression vector (GeneCopoeia, MD, USA) coding for six $\mathrm{N}$-terminally located histamine residues and expressed in BL21 (DE3) pLysS competent cells according to the manufacturer's instructions. The expressed recombinant 
protein was then purified over a $\mathrm{Ni}^{2+}$ affinity column using the AKTA Basic System (Amersham Biosciences) under denaturing conditions. Polyclonal antibodies were produced in a male New Zealand white rabbit by injecting $100 \mathrm{mg}$ of recombinant protein and boosting with onehalf of the initial amount at 2 and 3 weeks after the primary immunization. Reactivity of the pre-immune and immune sera was determined by ELISA.

\section{Real-time quantitative PCR (qPCR) analyses}

Total RNA of mice tissues was extracted using TRIzol reagent (Invitrogen) and was converted to cDNA using a RevertAid First-Strand cDNA Synthesis Kit (ThermoFisher). Real-time PCR was performed using SYBR Premix Ex Taq II (TaKaRa) on an iCycler RT-PCR Detection System (Bio-Rad Laboratories). The $\Delta \Delta C T$ method was used for data analysis. Each assay was performed in triplicate for each sample. The Gapdh gene was used as an internal control. The primers for real-time PCR are listed in Supplementary Table S5.

\section{Immunofluorescence (IF)}

For immunofluorescence assay, moue spermatozoa samples were fixed onto slides with $4 \%$ paraformaldehyde, permeabilized with $0.5 \%$ TritonX-100 in PBS, and blocked with $5 \%$ BSA. Next, The slides were sequentially incubated overnight at $4{ }^{\circ} \mathrm{C}$ with the following primary antibodies: anti-WDR63 (described above, 1:1000), antiWDR78 (sc-390633, Santa Cruz, 1: 50), anti-Acetyl- $\alpha-$ Tubulin (D20G3, CST, 1:1000). The slides were washed in 1× PBS, incubated with Alexa Fluor 488 (1:500, A21206, Thermo Fisher) or Alexa Fluor 594 (1:500, A11005, Thermo Fisher) labeled secondary antibodies for $2 \mathrm{~h}$ at room temperature, and then counterstained with Hoechst 33258 to label the nuclei. Images were acquired using a laser scanning confocal microscope (LS800).

\section{Coimmunoprecipitation (Co-IP)}

The extracted proteins were incubated with $3 \mu \mathrm{g}$ of target antibodies overnight at $4{ }^{\circ} \mathrm{C}$. Next, $50 \mu \mathrm{l}$ of Protein A/G magnetic beads (LSKMAGAG10, Millipore) was added to each incubation sample for $1 \mathrm{~h}$ at room temperature. The beads were washed three times with $1 \times$ PBS. Finally, the coimmunoprecipitated proteins were eluted by standard $1 \times$ SDS sample buffer and heated for $10 \mathrm{~min}$ at $70^{\circ} \mathrm{C}$ and then separated on $10 \%$ SDS-polyacrylamide gels and PVDF membranes for the immunoblot analysis.

\section{Histological analysis}

For H\&E (hematoxylin and eosin) staining, the epididymis was fixed in modified Davidson liquid (30\% of a $37-40 \%$ formaldehyde stock solution, 15\% ethanol, $5 \%$ glacial acetic acid, and $50 \%$ distilled water) and embedded in paraffin. Sections were cut at a $5 \mu \mathrm{m}$ thickness. The sections were then dewaxed with xylene, hydrated, $H \& E$ stained, dehydrated with ethanol $(70 \%, 80 \%, 90 \%, 100 \%)$ and finally blocked with resin. For periodic acid-Schiff (PAS) staining, testes are also fixed in MDF and embedded in paraffin. The sections were then deparaffinized, hydrated, stained with Periodic Acid-Schiff reagent, counterstained with hematoxylin, dehydrated and blocked.

\section{Immunoblot analysis}

The proteins of the cultured cells and mice testes tissues were extracted using a universal protein extraction lysis buffer (Bioteke) containing a protease inhibitor cocktail (Roche). The denatured proteins were separated on $10 \%$ SDS-polyacrylamide gels and transferred to a polyvinylidene difluoride (PVDF) membrane (Millipore) for the immunoblot analysis. The antibodies used in western blotting, Co-IP and immunofluorescence staining are as follows: anti-WDR63 (described above, 1:1000), anti-WDR78 (sc-390401, Santa Cruz, 1:100), anti-DNAH2 (HPA067103, SigmaAldrich, 1:1000), anti-DNAH10 (bs-11022R, Bioss, 1:1000), anti-HA-tag (M180-3, MBL, 1:1000), anti-Flagtag (F3165, Sigma-Aldrich, 1:1000).

\section{Scanning and transmission electron microscopy}

For scanning electron microscopy (SEM), the cauda epididymides obtained through dissection of adult male mice and incubated in human tubal fluid (HTF) medium (Millipore) supplemented with $10 \% \mathrm{FBS}$ at $37^{\circ} \mathrm{C}$ for 5 min. The sperms were fixed at $4{ }^{\circ} \mathrm{C}$ overnight with $2.5 \%$ glutaraldehyde in $0.2 \mathrm{M}$ cacodylate buffer $(50 \mathrm{mM}$ cacodylate, $50 \mathrm{mM} \mathrm{KCl}$, and $2.5 \mathrm{mM} \mathrm{MgCl} 2, \mathrm{pH} 7.2$ ). After immersed in $1 \% \mathrm{OsO}_{4}$ in $0.2 \mathrm{M}$ cacodylate buffer for $2 \mathrm{~h}$ at $4{ }^{\circ} \mathrm{C}$, the specimens were dehydrated through a graded ethanol series dried through the use of a $\mathrm{CO}_{2}$ critical-point dryer (Eiko HCP-2, Hitachi). Afterward, the specimens were mounted on aluminum stubs, sputter coated through using an ionic sprayer meter (Eiko E-1020, Hitachi), and analyzed via SEM (Stereoscan 260).

For transmission electron microscopy (TEM), the adult mouse cauda epididymis was fixed at $4{ }^{\circ} \mathrm{C}$ overnight with $2.5 \%$ glutaraldehyde in $0.2 \mathrm{M}$ cacodylate buffer. After washing in cacodylate buffer, the specimens were cut into small pieces of approximately $1 \mathrm{~mm}^{3}$ and immersed in $1 \%$ OsO4 in $0.2 \mathrm{M}$ cacodylate buffer for $2 \mathrm{~h}$ at $4{ }^{\circ} \mathrm{C}$. Next, the samples were washed and submerged in $0.5 \%$ uranyl acetate overnight, dehydrated through a graded ethanol series, and embedded in resin (Low Viscosity Embedding Media Spurr's Kit, EMS, 14300). Ultrathin sections were cut on an ultramicrotome and mounted on copper grids. The sections then were stained with uranyl acetate and lead citrate for $10 \mathrm{~min}$ and observed using a JEM-1400 transmission electron microscope (JEOL). 


\section{In vitro fertilization (IVF) and intracytoplasmic sperm injection (ICSI)}

Two-month-old B6D2F1 (C57BL/6 3 DBA2) wild-type female mice were superovulated by injecting 5-7.5 IU of pregnant mare serum gonadotropin (PMSG), followed by 5-7.5 IU of human chorionic gonadotropin (hCG) $48 \mathrm{~h}$ later. For IVF, wild-type and homozygous sperm samples were collected from mouse cauda epididymis incubated in human tubal fluid (HTF) medium drop. Then cumulusintact oocytes, collected from superovulated females, were transferred into the sperm-containing HTF drop. After 5-6h of incubation, the embryos were washed in HTF and transferred into KSOM medium (Millipore, Cat. \# MR-106-D) to further culture at $37^{\circ} \mathrm{C}$ under $5 \% \mathrm{CO}$. Fertilization rates were evaluated by recording the numbers of two-cell embryos and late-stage blastocysts at $20 \mathrm{~h}$ and $91 \mathrm{~h}$ later, respectively. For ICSI, oocytes were obtained from superovulated females, and sperm heads were injected into oocytes through using a Piezodriven pipette. Then the injected oocytes were cultured inKSOM medium at $37{ }^{\circ} \mathrm{C}$ under $5 \% \mathrm{CO} 2$. Two-cell embryos and blastocysts were counted $20 \mathrm{~h}$ and $96 \mathrm{~h}$ later, respectively.

\section{Statistical analysis}

Numerical data are presented as means \pm SEM. The statistical significance of the difference between the mean values for the different genotypes was examined using Student's $t$-test with a paired two-tailed distribution. The data were considered significant when $P \leq 0.05(*)$ and $0.01(* *)$.

\section{Acknowledgements}

The authors thank all the patients who provided samples to this research. This work was funded by the Program of the National Natural Science Foundation of China (31530047), the National Key Project of Research and Development Program (2018YFC1004200, 2018YFC1004202), the National Natural Science Foundation of China (81902836)

\section{Author details \\ ${ }^{1}$ Department of Epidemiology, School of Public Health, Southeast University, Nanjing, Jiangsu, China. ${ }^{2}$ State Key Laboratory of Reproductive Medicine, Nanjing Medical University, Nanjing, Jiangsu, China. ${ }^{3}$ Department of Epidemiology, Center for Global Health, School of Public Health, Nanjing Medical University, Nanjing, Jiangsu, China. ${ }^{4}$ State Key Laboratory of Reproductive Medicine, The Affiliated Suzhou Hospital of Nanjing Medical University, Suzhou, Jiangsu, China. ${ }^{5}$ Suzhou Municipal Hospital, Suzhou, Jiangsu, China. ${ }^{6}$ Institute of Reproductive and Stem Cell Engineering, School of Basic Medical Science, Central South University, Changsha, Hunan, China. ${ }^{7}$ Clinical Research Center for Reproduction and Genetics in Hunan Province, Reproductive and Genetic Hospital of CITIC-Xiangya, Changsha, Hunan, China. ${ }^{8}$ Center of Reproductive Medicine, First Affiliated Hospital of Nanjing Medical University, Nanjing, Jiangsu, China}

\section{Author contributions}

S.L., Y.Y.G., Y.F.W., and Z.B.H. designed experiments and collected data. G.Y.Y., T. J., C.W., and Z.B.H. contributed to data analyses of WES. S.L., Y.Y.G., Z.B.H. wrote the manuscript. S.L., Y.F.W., Y.L., L.L.M., X.Y.Y., and Y.Q.T. collected the blood, semen and testicular tissue samples and the volunteers' clinical information. $S$. L., Y.F.W., W.W.Y. performed the animal experiments. S.L. and M.X.L. carried out the electron microscopy experiments. S.L., C.M.J.L., W.W.Y. contributed to the CASA analyses. S.L., C.M.J.L., W.W.Y. performed the PCR-sequencing analyses and Sanger sequencing. X.Z. contributed to generation of Wdr63 knockout mouse models. S.L. and M.X.L. carried out the WDR63 combinant protein and antibody generation. H.B.S. S.M.Y., X.Y.Y., X.J.G., and Z.B.H. supervised the study and wrote the manuscript.

\section{Data availability}

All relevant data are available from the corresponding author. Source data are provided with this paper.

\section{Competing interests}

The authors declare no competing interests.

\section{Publisher's note}

Springer Nature remains neutral with regard to jurisdictional claims in published maps and institutional affiliations.

Supplementary information The online version contains supplementary material available at https://doi.org/10.1038/s41421-021-00327-5.

Received: 14 April 2021 Accepted: 19 August 2021

Published online: 16 November 2021

\section{References}

1. Zhu, Z. et al. Spermatogenesis is normal in Tex33 knockout mice. Peer J. 8 e9629 (2020).

2. Agarwal, A., Mulgund, A., Hamada, A. \& Chyatte, M. R. A unique view on male infertility around the globe. Reprod. Biol. Endocrinol. 13, 37 (2015).

3. Tuttelmann, F., Ruckert, C. \& Ropke, A. Disorders of spermatogenesis: perspectives for novel genetic diagnostics after 20 years of unchanged routine. Med. Genet. 30, 12-20 (2018).

4. Feng, T. et al. Eef2k is not required for fertility in male mice. Transl. Androl. Urol. 10, 1988-1999 (2021).

5. Toure, A. et al. The genetic architecture of morphological abnormalities of the sperm tail. Hum. Genet. 140, 21-42 (2021).

6. Liu, C. et al. Bi-allelic DNAH8 variants lead to multiple morphological abnormalities of the sperm flagella and primary male infertility. Am. J. Hum. Genet. 107, 330-341 (2020).

7. He, X. et al. Bi-allelic loss-of-function variants in CFAP58 cause flagellar axoneme and mitochondrial sheath defects and asthenoteratozoospermia in humans and mice. Am. J. Hum. Genet. 107, 514-526 (2020).

8. Wang, M. et al. Single-cell RNA sequencing analysis reveals sequential cell fate transition during human spermatogenesis. Cell Stem Cell 23, 599-614 e594 (2018).

9. Krausz, C. \& Riera-Escamilla, A. Genetics of male infertility. Nat. Rev. Urol. 15, 369-384 (2018)

10. Cramer, P. Organization and regulation of gene transcription. Nature $\mathbf{5 7 3}$ 45-54 (2019).

11. Liu, W. et al. Bi-allelic mutations in TTC21A induce asthenoteratospermia in humans and mice. Am. J. Hum. Genet. 104, 738-748 (2019).

12. Shen, $\mathrm{Y}$. et al. Loss-of-function mutations in $\mathrm{QRICH} 2$ cause male infertility with multiple morphological abnormalities of the sperm flagella. Nat. Commun. 10 433 (2019).

13. Coutton, C. et al. Bi-allelic mutations in ARMC2 lead to severe asthenoteratozoospermia due to sperm flagellum malformations in humans and mice. Am. J. Hum. Genet. 104, 331-340 (2019).

14. Tu, C. et al. Bi-allelic mutations of DNAH10 cause primary male infertility with asthenoteratozoospermia in humans and mice. Am. J. Hum. Genet. 108, 1466-1477 (2021)

15. Sironen, A., Shoemark, A., Patel, M., Loebinger, M. R. \& Mitchison, H. M. Sperm defects in primary ciliary dyskinesia and related causes of male infertility. Cell Mol. Life Sci. 77, 2029-2048 (2020).

16. Viswanadha, R., Sale, W. S. \& Porter, M. E. Ciliary motility: regulation of axonemal dynein motors. Cold Spring Harb. Perspect. Biol. 9, a018325 (2017).

17. Zhang, Y. et al. Vertebrate Dynein-f depends on Wdr78 for axonemal localization and is essential for ciliary beat. J. Mol. Cell Biol. 11, 383-394 (2019).

18. Ben Khelifa, M. et al. Mutations in DNAH1, which encodes an inner arm heavy chain dynein, lead to male infertility from multiple morphological abnormalities of the sperm flagella. Am. J. Hum. Genet. 94, 95-104 (2014). 
19. Cao, X. W., Lin, K., Li, C. Y. \& Yuan, C. W. A review of WHO Laboratory Manual for the Examination and Processing of Human Semen (5th edition). Zhonghua nan ke xue = Natl J. Androl. 17, 1059-1063 (2011)

20. Bui, K. H., Sakakibara, H., Movassagh, T., Oiwa, K. \& Ishikawa, T. Molecular architecture of inner dynein arms in situ in Chlamydomonas reinhardtii flagella. J. Cell Biol. 183, 923-932 (2008).

21. Du, G. et al. mRBPome capture identifies the RNA-binding protein TRIM71, an essential regulator of spermatogonial differentiation. Development 147, dev184655 (2020).

22. Wang, $X$. et al. The heat shock protein family gene Hspa1l in male mice is dispensable for fertility. PeerJ. 8, e8702 (2020).

23. Tharakan, T., Luo, R., Jayasena, C. N. \& Minhas, S. Non-obstructive azoospermia: current and future perspectives. Fac. Rev. 10, 7 (2021).

24. Coutton, C. et al. Mutations in CFAP43 and CFAP44 cause male infertility and flagellum defects in Trypanosoma and human. Nat. Commun. 9, 686 (2018).

25. Tang, S. et al. Biallelic mutations in CFAP43 and CFAP44 cause male infertility with multiple morphological abnormalities of the sperm flagella. Am. J. Hum. Genet. 100, 854-864 (2017).

26. Kherraf, Z. E. et al. A Homozygous ancestral sva-insertion-mediated deletion in WDR66 induces multiple morphological abnormalities of the sperm flagellum and male infertility. Am. J. Hum. Genet. 103, 400-412 (2018).

27. Young, S. A. et al. CRISPR/Cas9-mediated rapid generation of multiple mouse lines identified Ccdc63 as essential for spermiogenesis. Int. J. Mol. Sci. 16, 24732-24750 (2015)

28. Hamada, Y., Tsurumi, Y., Nozaki, S., Katoh, Y. \& Nakayama, K. Interaction of WDR60 intermediate chain with TCTEX1D2 light chain of the dynein-2 complex is crucial for ciliary protein trafficking. Mol. Biol. Cell 29, 1628-1639 (2018).

29. Tu, C. et al. Identification of DNAH6 mutations in infertile men with multiple morphological abnormalities of the sperm flagella. Sci. Rep. 9, 15864 (2019).

30. Perrone, C. A., Yang, P., OToole, E., Sale, W. S. \& Porter, M. E. The Chlamydomonas IDA7 locus encodes a 140-kDa dynein intermediate chain required to assemble the I1 inner arm complex. Mol. Biol. cell 9, 3351-3365 (1998).

31. Yang, P. \& Sale, W. S. The Mr 140,000 intermediate chain of Chlamydomonas flagellar inner arm dynein is a WD-repeat protein implicated in dynein arm anchoring. Mol. Biol. Cell 9, 3335-3349 (1998).
32. Wang, K., Li, M. \& Hakonarson, H. ANNOVAR: functional annotation of genetic variants from high-throughput sequencing data. Nucleic Acids Res. 38, e164 (2010).

33. Liu, C. et al. Bi-allelic DNAH8 Variants Lead to Multiple Morphological Abnormalities of the Sperm Flagella and Primary Male Infertility. Am. J. Hum. Genet. https://doi.org/10.1016/j.ajhg.2020.06.004 (2020).

34. Liu, C. et al. Bi-allelic mutations in TTC29 cause male subfertility with asthenoteratospermia in humans and mice. Am. J. Hum. Genet. 105, 1168-1181 (2019).

35. Cooke, H. J. \& Saunders, P. T. Germ cell transplantation-a fertile field. Nat. Med. 6, 16-17 (2000).

36. Hua, J. T. et al. Risk SNP-mediated promoter-enhancer switching drives prostate cancer through IncRNA PCAT19. Cell 174, 564-575 e518 (2018).

37. Janaka, S. K. \& Evans, D. T. A SNP of IncRNA gives HIV-1 a boost. Nat. Immunol. 20, 778-780 (2019).

38. Kumar, P., Henikoff, S. \& Ng, P. C. Predicting the effects of coding nonsynonymous variants on protein function using the SIFT algorithm. Nat. Protoc. 4, 1073-1081 (2009)

39. Adzhubei, I. A. et al. A method and server for predicting damaging missense mutations. Nat. Methods 7, 248-249 (2010).

40. Schwarz, J. M., Cooper, D. N., Schuelke, M. \& Seelow, D. MutationTaster2: mutation prediction for the deep-sequencing age. Nat. Methods 11, 361-362 (2014).

41. Wang, C. et al. Systematic identification of genes with a cancer-testis expression pattern in 19 cancer types. Nat. Commun. 7, 10499 (2016).

42. Martinez, G. et al. Whole-exome sequencing identifies mutations in FSIP2 as a recurrent cause of multiple morphological abnormalities of the sperm flagella. Hum. Reprod. 33, 1973-1984 (2018).

43. Amiri-Yekta, A. et al. Whole-exome sequencing of familial cases of multiple morphological abnormalities of the sperm flagella (MMAF) reveals new DNAH1 mutations. Hum. Reprod. 31, 2872-2880 (2016).

44. Li, Y. et al. DNAH2 is a novel candidate gene associated with multiple morphological abnormalities of the sperm flagella. Clin. Genet. 95, 590-600 (2019).

45. Hon, C. C. et al. An atlas of human long non-coding RNAs with accurate $5^{\prime}$ ends. Nature 543, 199-204 (2017). 